1 Ministério da Saúde, Departamento de Atenção Básica - Brasília (DF), Brasil.

pauline.cavalcanti@gmail. com

2 Ministério da Saúde, Secretaria de Gestão do Trabalho e da Educação na Saúde - Brasília (DF) Brasil.

aristidesoliveira80@gmail. com

3 Universidade de Brasília (UnB), Faculdade de Ciências da Saúde (FS), Departamento de Saúde Coletiva (DSC) - Brasílía (DF), Brasil.

fatimasousa@unb.br

\section{Quais são os desafios para a qualificação da Atenção Básica na visão dos gestores municipais?}

\author{
What are the challenges for the qualification of Primary Care in the \\ view of municipal managers?
}

Pauline Cristine da Silva Cavalcanti $\mathbf{1}$, Aristides Vitorino de Oliveira Neto ${ }^{2}$, Maria Fátima de Sousa ${ }^{\mathbf{3}}$

RESUMO Nos últimos anos, a Atenção Básica proporcionou muitos avanços na saúde da população, porém, muitos desafios ainda persistem. Este trabalho busca analisar os principais desafios da Atenção Básica na visão dos gestores municipais de saúde, identificando e analisando descritivamente os cinco mais frequentes nos âmbitos nacional, regional e por estrato de municípios. Realizou-se uma abordagem quantitativa com análise de dados secundários da Atenção Básica à saúde, oriundos das bases de dados do Ministério da Saúde (MS). Observouse que muitos desafios são recorrentes e outros evidenciados em alguma região ou estrato, sugerindo sua dimensão no contexto local.

PALAVRAS-CHAVE Atenção Primária à Saúde; Saúde da família; Avaliação em Saúde.

ABSTRACT In the last years, the Primary Care has provided many advances in the community health, however, many challenges still remain. This paper seeks to analyze the main challenges of Primary Care in the view of the municipal health managers, identifying and analyzing descriptively the five most common ones at the levels national, regional and by stratum of municipalities. It was held a quantitative approach with the analysis of secondary data of the Primary Health Care, derived from the databases of the Ministry of Health. It was noted that many challenges are recurrent and others are shown in some specific region or stratum, suggesting their dimension in the local context.

KEYWORDS Primary Health Care; Family health; Health evaluation. 


\section{Introdução}

Nos últimos anos, a Atenção Básica (AB) tem recebido muito destaque no SUS, especialmente a partir da criação do Programa de Agentes Comunitários de Saúde (Pacs), em 1992, e do Programa Saúde da Família (PSF), em 1994. O termo Programa Saúde da Família, adotado na sua implantação, se deu em função da necessidade de criar uma marca capaz de dialogar com a matriz orçamentária do Ministério da Saúde, que, à época, estruturava suas ações por programa. Entretanto, o desenho dos seus objetivos e diretrizes orientavam para a adoção de uma estratégia capaz de induzir mudanças no modelo de atenção à saúde (sousA, 2002, 2003A, 2003B).

Conforme se deram sua expansão em base municipal e seus efetivos resultados, o termo Estratégia Saúde da Família (ESF) ganhou maior relevância. Dessa forma, adquiriu a posição de estratégia prioritária nos processos organizativos da $\mathrm{AB}$ (sousA, 2002, 2003A; SOUSA; MENDES, 2003). Atualmente, a ESF é o principal investimento do MS para promover a (re)organização das ações de $\mathrm{AB}$ visando à consolidação do Sistema Único de Saúde (SUS) no País (FaUsto; MENDOnÇA; GIOVANELLA, 2013; PAIM ET AL., 2001; TANAKA; RIBEIRO, 2009; ROCHA ET AL., 2008; SOUSA, 2007).

A AB cumpre o papel estratégico no SUS de garantir a universalidade do acesso, a cobertura universal e a efetivação da integralidade (PAIM ET AL., 2011; BRASIL, 2012; BRASIL, 2011B; TANAKA, RIBEIRO, 2009). A atual Política Nacional de Atenção Básica (PNAB) define que a AB abrange um conjunto de ações relacionadas com aspectos coletivos e individuais de uma população, envolvendo a promoção e a proteção da saúde, a prevenção de agravos, o diagnóstico, o tratamento, a reabilitação e a manutenção da saúde, com objetivo de desenvolver uma atenção integral que impacte a situação de saúde dos indivíduos.

A $\mathrm{AB}$ deve levar ações de atenção à saúde o mais próximo possível das pessoas, buscando resolver os problemas de saúde mais frequentes e de maior relevância para a população (BRASIL, 2011A). Deve, ainda, ser a porta de entrada preferencial do SUS (BRASIL, 2011; TANAKA; RIBEIRO, 2009) para novas necessidades e problemas (STARFIELD, 2002).

Desde a criação do SUS e, posteriormente, do PSF, houve considerável expansão da cobertura dos serviços de $\mathrm{AB}$, aumentando o acesso a ações de saúde para uma grande parte da população brasileira. Em consequência disso, houve melhoria significativa em muitos resultados em saúde do País (PAIM ET AL., 2011). No entanto, assim como o SUS, pode-se dizer que a $A B$ enfrenta desafios 'antigos', 'contemporâneos' (SOUSA, 2007) e 'futuros' (PAIM, ET AL., 2011), que remetem a problemas importantes e complexos que devem ser resolvidos progressivamente para permitir maiores avanços em direção à sua qualificação.

O objetivo deste estudo é analisar os principais desafios da $\mathrm{AB}$ na perspectiva dos gestores municipais de saúde. Nesse contexto, busca identificar os cinco principais desafios apontados pelos gestores da $\mathrm{AB}$ na etapa de adesão ao Programa Nacional de Melhoria do Acesso e da Qualidade da Atenção Básica (PMAQ) e realizar análise descritiva dos desafios mais frequentes nacionalmente, bem como por região e estrato do País.

\section{Os desafios da Atenção Básica à saúde}

O SUS enfrenta muitos desafios que demandam ações governamentais em busca de caminhos que possam levar a mudanças na organização do sistema e dos serviços de saúde e na forma de cuidar dos usuários. No entanto, é preciso investir em ações transformadoras para interferir no modelo constituído (MERHY; MALTA; SANTOS, 2004).

Considerando que a ESF está efetivamente incorporada à $\mathrm{AB}$ e que tem função de reordenar o SUS e organizar os serviços de saúde nos municípios (PAIM ET AL., 2011; TANAKA; 
RIBEIRO, 2009; CAETANO; DAIN, 2002), entende-se que os desafios colocados para o SUS são convergentes com aqueles que permeiam a $\mathrm{AB} / \mathrm{ESF}$.

De acordo com o 'Triângulo de Governo', de Carlos Matus, para que os desafios da $A B$ sejam enfrentados, é fundamental que a $\mathrm{AB}$ entre na agenda prioritária dos gestores, isto é, integre o plano de governo. É necessário, ainda, que os gestores aprofundem o conhecimento sobre as tensões, disputas, atores e recursos que permeiam a $A B$, de modo que consigam buscar graus elevados de governabilidade. Por outro lado, tomar os desafios da AB como agenda estratégica para reordenar o SUS exige e, ao mesmo tempo, possibilita o desenvolvimento de uma capacidade de governo, ou seja, de "um conjunto de técnicas, métodos, destrezas e habilidades de um gestor e de sua equipe para atingir os objetivos declarados" (MATUS, 1993, P. 61 APUD TEIXEIRA, 2010).

O campo da saúde é construído a partir de tensões permanentes, que podem servir de alavancas e serem exploradas como potência para ações transformadoras. Pode-se dizer que o território da saúde é composto por 'terrenos minados', que nos forçam a agir ou permitem desenhar arranjos organizacionais para construir modos de produzir saúde. Mesmo quando há a “construção de um certo modo de agir em saúde, de um certo modelo de organizar os processos de trabalho, de uma certa forma de produzir o cuidado", as tensões permanentes não desaparecem. Dessa forma, as tensões podem servir como impulso para interferir no modelo constituído ou para manter o modelo dado (MERHY; MALTA; SANTOS, 2004).

Diversos estudos (ROCHA ET AL., 2008; PAIM ET AL., 2011; CAETANO; DAIN, 2002) apontam os avanços da $\mathrm{AB}$, principalmente a partir dos resultados alcançados pelo PSF e, mais atualmente, pela ESF. Entretanto, a saúde ainda sofre com questões que dificultam a superação dos seus desafios. De acordo com Paim et al. (2011), os desafios enfrentados pelos SUS são, na verdade, políticos. Assim, só serão resolvidos a partir dos esforços conjuntos dos indivíduos e da sociedade com a intenção de garantir ao sistema sua sustentabilidade política, econômica, científica e tecnológica.

Um bom exemplo para refletir sobre os problemas políticos do sistema de saúde é a questão do financiamento da $\mathrm{AB}$, que, de acordo com Sousa (2008), impõe novos desafios, principalmente no que diz respeito à prática 'cooperativa' e 'complementar' construída entre os gestores do SUS, ressaltando que as esferas de governo (federal, estadual e municipal) devem "estabelecer pactos de corresponsabilidade no financiamento das ações e dos serviços de saúde, provendo o direito à saúde de forma responsável e solidária”.

Neste sentido, Paim et al. (2011) explicam que, para o SUS superar os desafios atuais, é necessária maior mobilização política atuando no sentido de reestruturar o financiamento das ações de saúde e de redefinir os papéis dos setores público e privado. Segundo os autores, os desafios futuros do SUS estão relacionados aos citados anteriormente e a outros voltados à adequação do modelo de atenção, para atender às necessidades da população e do País, e à promoção da qualidade do cuidado e da segurança dos pacientes.

Outro problema relevante para a $\mathrm{AB}$ refere-se à questão dos recursos humanos disponíveis para atuar na saúde da família, considerando que há escassez e insuficiência de profissionais, despreparo e precária qualificação destes (CAETANO; DAIN, 2002) para atender às exigências desse modelo de atenção, que envolve uma diversificada e complexa realidade sanitária (CAETANO; DAIN, 2002; CAMELO; ANGERAMI, 2008). Tal modelo requer dos profissionais conhecimentos e habilidades específicas para trabalharem junto às comunidades, além de criatividade e adequado suporte técnico-científico (CAMELO; ANGERAMI, 2008).

Para Rocha et al. (2008), as soluções devem ser buscadas para melhoria do atendimento 
em serviços de $\mathrm{AB}$ e focar desde aspectos relacionados ao atendimento clínico-assistencial até o reconhecimento e incentivo a experiências exitosas que levam à reorganização da AB. A busca da qualidade não envolve apenas mudanças nas práticas dos profissionais das equipes. Ela exige, também, a qualificação da gestão, especialmente no nível local, sobretudo no que diz respeito às suas práticas e posturas (SAVASSI, 2012).

Savassi (2012) aponta alguns aspectos que devem ser foco da atuação dos gestores visando à melhoria da qualidade na $\mathrm{AB}$, como: a ambiência das unidades de saúde; o uso e a disponibilidade de tecnologias adequadas ao trabalho; passar uma imagem de confiabilidade e competência técnica dos profissionais das equipes; ter agilidade no atendimento; a definição das funções e obrigações dos setores e funcionários; e a priorização da satisfação do usuário.

Em síntese, podemos dizer que os desafios da $A B$ são um problema cotidiano para gestores, profissionais das Equipes de Atenção Básica (EqAB) e, mais ainda, para os usuários. Embora exista uma crescente conscientização sobre a importância da qualidade da atenção à saúde no Brasil, ainda é necessário avançar muito para assegurar padrões elevados (PAIM ET AL., 2011).

Algumas iniciativas vêm sendo desenvolvidas visando reconhecer e superar os problemas da $\mathrm{AB}$, principalmente após a criação do PSF (ROCHA ET AL., 2008; PAIM ET AL., 2011). Os atuais desafios da $\mathrm{AB}$ imprimem a necessidade de desenvolver e aperfeiçoar as inciativas com vistas à superação dos seus problemas e com o compromisso direcionado à melhoria da qualidade na $\mathrm{AB}$ (PAIM ET AL., 2011; CAETANO; DAIN, 2002).

Logo no início de 2011, após várias reuniões, o governo federal destacou a $\mathrm{AB}$ como prioridade para a saúde e determinou a criação de um conjunto de programas para tal. A partir disso, sugiram os desafios para o desenvolvimento da $\mathrm{AB}$, que deveriam ser foco de atuação por meio de ações e programas da nova Política Nacional de Atenção Básica (PNAB) (PINTO; SOUSA; FERLA, 2014).

De acordo com Pinto (2011) apud Pinto, Sousa e Ferla (2014, P. 360), os desafios para a $\mathrm{AB}$ no Brasil são:

1 - Financiamento insuficiente da Atenção Básica; 2 - Infraestrutura das UBS inadequada; 3 - Baixa informatização dos serviços e pouco uso das informações disponíveis para a tomada de decisões na gestão e a atenção à saúde; 4 - Necessidade de ampliar o acesso, reduzindo tempos de espera e garantindo atenção, em especial, para grupos mais vulneráveis; 5 - Necessidade de melhorar a qualidade dos serviços incluindo acolhimento, resolubilidade e longitudinalidade do cuidado; 6 - Pouca atuação na promoção da saúde e no desenvolvimento de ações intersetoriais; 7 - Desafio de avançar na mudança do modelo de atenção e na mudança de modelo e qualificação da gestão; 8 - Inadequadas condições e relações de trabalho, mercado de trabalho predatório, déficit de provimento de profissionais e contexto de baixo investimento nos trabalhadores; 9 - Necessidade de contar com profissionais preparados, motivados e com formação específica para atuação na Atenção Básica; 10 - Importância de ampliar a legitimidade da Atenção Básica junto aos usuários e de estimular a participação da sociedade.

A partir da compreensão de que esses são os desafios a serem enfrentados na $A B$, buscou-se promover discussões e construir consenso acerca desse entendimento nas esferas de pactuação tripartite do SUS. Nesse contexto, em abril do mesmo ano, o Conselho Nacional de Saúde (CNS) aprovou a Resolução $\mathrm{n}^{\circ} 439$ sobre a $\mathrm{AB}$, que serviu para revisão da PNAB (PINTO; SOUSA; FERLA, 2014).

Na Resolução, o CNS ressalta que as ações de $\mathrm{AB}$ são de responsabilidade das três esferas de gestão do SUS e aponta a necessidade de: "aumentar a destinação de recursos financeiros para a Atenção Básica”; garantir ações 
necessárias para que a Rede de Atenção Básica se efetive como a "principal porta de entrada do SUS"; "fortalecer o controle social, a participação da comunidade em cada serviço", realizar "coletas sistemáticas da opinião e satisfação do usuário"; "garantir profissionais de saúde em todas as regiões e localidades do País"; que o MS e as Secretarias Estaduais e Municipais "aprimorem e qualifiquem os mecanismos de controle, fiscalização do cumprimento de responsabilidades [...] e avaliação da qualidade dos serviços de Atenção Básica” (BRASIL, 2011B).

A nova PNAB engloba diversas iniciativas que dialogam com os desafios apontados pelo governo federal e com as propostas do CNS. Neste contexto, destacam-se: o Programa de Requalificação das Unidades Básicas de Saúde (UBS); a criação do novo Sistema de Informação da Atenção Básica e a estratégia e-SUS AB; o Programa Telessaúde Brasil Redes; o Programa Nacional de Melhoria do Acesso e da Qualidade da Atenção Básica (PMAQ); a reestruturação do Programa Saúde na Escola; a criação do Programa Academia da Saúde; a nova Política Nacional de Alimentação e Nutrição; a Política de Educação Permanente do SUS; o Plano Nacional de Educação Médica; o Programa de Valorização e Atenção Básica e o Programa Mais Médicos, além de medidas para adequação do financiamento da $\mathrm{AB}$ (PINTO; SOUSA; FERLA, 2014).

\section{Metodologia}

Trata-se de estudo quantitativo de análise de dados secundários referentes à Atenção Básica em saúde, oriundos das bases de dados do Ministério da Saúde/Secretaria de Atenção à Saúde/Departamento de Atenção Básica (DAB). Analisou-se a base de dados do Sistema do Programa Nacional de Melhoria do Acesso e da Qualidade da Atenção Básica visando a identificar os principais desafios para a qualificação da $\mathrm{AB}$ no Brasil.

Para este estudo, foram utilizadas informações de 3.934 municípios das 27 Unidades Federativas do País, que selecionaram, entre os vinte e cinco desafios apontados pelo MS (quadro 1), os cinco principais desafios para a qualificação da $\mathrm{AB}$ no seu município no momento de adesão ao PMAQ, em 2011.

\begin{tabular}{ll}
\hline Quadro 1. Desafios para a qualificação da AB municipal - 2011 \\
\hline Desafios & \multicolumn{1}{c}{ Descrição } \\
\hline 1 & Aperfeiçoar a capacidade de gestão de pessoas da Coordenação da ABS \\
2 & Aperfeiçoar a capacidade de gestão financeira da Coordenação da ABS \\
3 & Aperfeiçoar a capacidade de gestão de equipamentos e insumos da Coordenação da ABS \\
4 & Aperfeiçoar os processos de monitoramento e avaliação da ABS com base em indicadores \\
5 & Auxiliar as equipes na análise e no manejo de informaçães em saúde, inclusive indicadores, de modo que \\
6 & tenham valor de uso e sirvam para a tomada de decisão no âmbito local \\
7 & Qualificar a equipe de gestão municipal para realização de apoio institucional \\
8 & Compor grupo de apoiadores das equipes de saúde \\
9 & Estimular a gestão colegiada nas unidades \\
10 & Estimular a constituição de espaços regulares de reunião das equipes para discussão do processo de \\
11 & trabalho e construção/acompanhamento de projetos terapêuticos singulares \\
12 & Realizar reformas das unidades próprias existentes \\
13 & Realizar construção de unidades em substituição de unidade alugadas \\
\hline
\end{tabular}




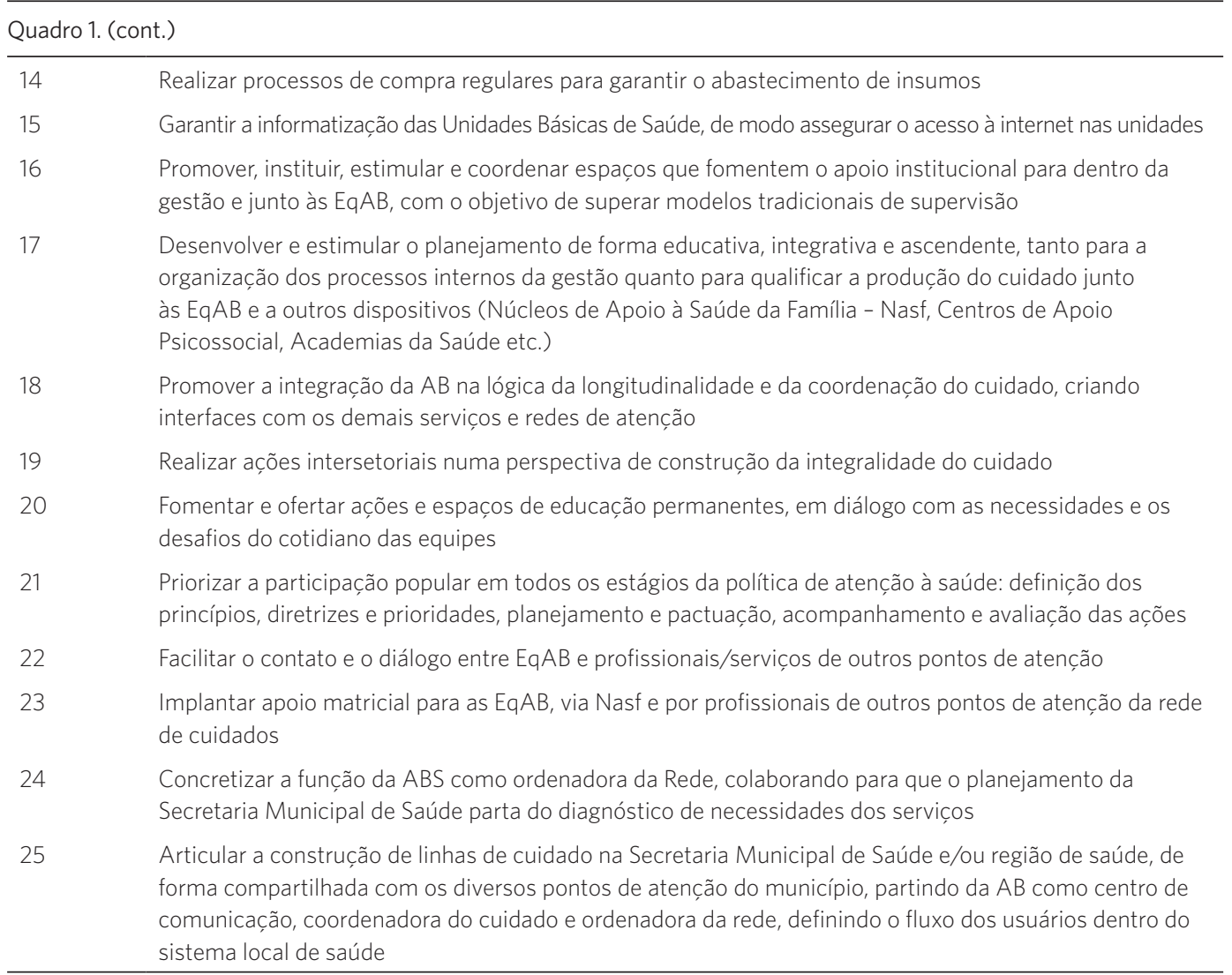

Fonte: Elaboração própria

Para as análises dos dados da adesão ao PMAQ, utilizou-se o programa Microsoft Office Excel 2010 para Windows $^{\circledR}$. As variáveis selecionadas para este estudo foram armazenadas em um banco de dados estruturado. Em seguida, foram calculadas as frequências absoluta e relativa para os vinte e cinco desafios e criadas tabelas, permitindo identificar os cinco mais frequentes, considerando os agregados Brasil, Regiões do País e Estratos dos municípios.

Como este estudo utiliza dados secundários do Ministério da Saúde, está dispensado de aprovação pelo Comitê de Ética. Os dados foram solicitados formalmente ao DAB/MS.

\section{Estratos do PMAQ}

Em 2011, o Ministério da Saúde lançou o Programa Nacional de Melhoria do Acesso e da Qualidade da Atenção Básica com o objetivo de melhorar o acesso e a qualidade dos serviços de saúde da Atenção Básica. O Programa busca avaliar as EqAB - por meio da verificação de padrões que expressem o acesso e a qualidade do serviço - e o desenvolvimento de ações dos gestores da $\mathrm{AB}$, com vistas à melhoria dos serviços do município.

O PMAQ propõe o desenvolvimento de diversas ações, desde a adesão das equipes, passando por monitoramento de indicadores e implantação de processos autoavaliativos até a avaliação externa. Ao final desse processo, é possível conhecer o resultado de cada equipe participante do Programa. No PMAQ, os resultados das equipes de um município são comparados com os resultados das equipes de outros municípios que compõem esse mesmo estrato.

Considerando que a diversidade de cenários socioeconômicos, epidemiológicos e demográficos entre os municípios 
brasileiros pode produzir diferenças entre os municípios e em seus sistemas locais de saúde e suas EqAB, o MS criou os 'Estratos', com o objetivo de garantir maior equidade no processo de comparação dos resultados alcançados pelas EqAB no Programa (BRASIL, 2012B).

Os estratos foram definidos a partir do cálculo de um índice municipal - que varia de zero a dez, com base em cinco indicadores (Produto Interno Bruto per Capita, Percentual da população com plano de saúde, Percentual da população com Bolsa Família, Percentual da população em extrema pobreza e Densidade demográfica) - e do porte populacional do município (BRASIL, 2012B). A seguir, estão descritos os seis estratos nos quais estão distribuídos os municípios do País:

- Estrato 1 - composto por municípios com índice menor que 4,82 e população de até 10 mil habitantes;

- Estrato 2 - composto por municípios com índice menor que 4,82 e população de até 20 mil habitantes;

- Estrato 3 - composto por municípios com índice menor que 4,82 e população de até 50 mil habitantes;
- Estrato 4 - composto por municípios com índice entre 4,82 e 5,4 e população de até 100 mil habitantes; e municípios com pontuação menor que 4,82 e população entre 50 e 100 mil habitantes;

- Estrato 5 - Municípios com índice entre 5,4 e 5,85 e população de até 500 mil habitantes; e municípios com pontuação menor que 5,4 e população entre 100 e 500 mil habitantes; e

- Estrato 6 - Municípios com população acima de 500 mil habitantes ou com pontuação igual ou superior a 5,85.

\section{Resultados e discussão}

Nesta seção, mostraremos os principais desafios para a qualificação da $\mathrm{AB}$ na perspectiva dos gestores municipais, considerando o nível Nacional, as Regiões do País e os Estratos dos municípios.

Ao final da adesão, em 2011, os gestores de 3.934 municípios do País selecionaram os principais desafios para a qualificação da $\mathrm{AB}$ municipal. A tabela 1 a seguir apresenta a classificação dos desafios de acordo com as frequências absoluta e relativa, calculadas para cada um deles.

Tabela 1. Classificação dos desafios para a qualificação da AB nos municípios em nível Nacional - 2011

\begin{tabular}{lrrr}
\hline Desafios & Frequência absoluta (№) & Frequência relativa (\%) & Classificação do desafio (C) \\
\hline 4 & $\mathbf{1 . 7 6 0}$ & 9,8 & $\mathbf{1 0}$ \\
5 & $\mathbf{1 . 6 0 2}$ & 8,9 & $\mathbf{2}=$ \\
1 & $\mathbf{1 . 2 6 6}$ & 7,1 & $\mathbf{3}$ \\
15 & $\mathbf{1 . 1 1 3}$ & 6,2 & $\mathbf{4}=$ \\
10 & $\mathbf{1 . 0 6 1}$ & 5,9 & $\mathbf{5 0}$ \\
25 & 967 & 5,4 & 60 \\
17 & 949 & 5,3 & 70 \\
13 & 867 & 4,8 & 80 \\
24 & 799 & 4,5 & 90 \\
\hline
\end{tabular}




\begin{tabular}{|c|c|c|c|}
\hline \multicolumn{4}{|c|}{ Tabela 1. (cont.) } \\
\hline 9 & 732 & 4,1 & 10 은 \\
\hline 2 & 612 & 3,4 & 110 \\
\hline 14 & 597 & 3,3 & $12^{\circ}$ \\
\hline 21 & 552 & 3,1 & $13 ㅇ$ \\
\hline 22 & 552 & 3,1 & $14^{\circ}$ \\
\hline 20 & 546 & 3,0 & $15 \circ$ \\
\hline 19 & 530 & 3,0 & $16^{\circ}$ \\
\hline 11 & 493 & 2,7 & $17^{\circ}$ \\
\hline 3 & 490 & 2,7 & $18^{\circ}$ \\
\hline 6 & 474 & 2,6 & 19 은 \\
\hline 18 & 474 & 2,6 & $20^{\circ}$ \\
\hline 12 & 413 & 2,3 & $21^{\circ}$ \\
\hline 16 & 350 & 2,0 & $22^{\circ}$ \\
\hline 23 & 299 & 1,7 & 23 \\
\hline 7 & 276 & 1,5 & $24 \stackrel{\circ}{-}$ \\
\hline 8 & 193 & 1,1 & $25^{\circ}$ \\
\hline Total & 17.967 & 100,0 & - \\
\hline
\end{tabular}

Fonte: Elaboração da própria

Conforme a tabela 1, os cinco desafios desafio 4, desafio 5, desafio 1, desafio 15 e para a qualificação da $\mathrm{AB}$ nos municípios, desafio 10 (ver descrição dos desafios no considerando o agregado Nacional, são: quadro 1).

\begin{tabular}{|c|c|c|c|c|c|c|c|c|c|c|c|c|c|c|c|}
\hline \multirow{2}{*}{ Desafios } & \multicolumn{3}{|c|}{ Centro-oeste } & \multicolumn{3}{|c|}{ Nordeste } & \multicolumn{3}{|c|}{ Norte } & \multicolumn{3}{|c|}{ Sudeste } & \multicolumn{3}{|c|}{ Sul } \\
\hline & № & $\%$ & C & № & $\%$ & C & № & $\%$ & C & № & $\%$ & C & № & $\%$ & C \\
\hline 1 & 99 & 5,6 & 60 & 389 & 6,7 & 3음 & 102 & 8,0 & 3음 & 373 & 6,9 & 3ㅇ & 304 & 7,6 & 3음 \\
\hline 2 & 53 & 3,0 & $17^{\circ}$ & 192 & 3,3 & $13 \circ$ & 62 & 4,9 & 70 & 185 & 3,4 & $11^{\circ}$ & 122 & 3,0 & $16^{\circ}$ \\
\hline 3 & 39 & 2,2 & 23 & 184 & 3,2 & $14 \div$ & 44 & 3,4 & $13 \circ$ & 140 & 2,6 & 190 & 86 & 2,1 & $20 \circ$ \\
\hline 4 & 146 & 8,2 & 10 & 585 & 10,1 & 10 & 135 & 10,6 & 10 & 533 & 9,8 & 10 & 365 & 9,1 & 20 \\
\hline 5 & 126 & 7,1 & 20 & 511 & 8,8 & 20 & 117 & 9,2 & $2^{\circ}$ & 483 & 8,9 & 20 & 370 & 9,2 & 10 \\
\hline 6 & 44 & 2,5 & $22^{\circ}$ & 167 & 2,9 & $17^{\circ}$ & 40 & 3,1 & $14^{\circ}$ & 134 & 2,5 & $20^{\circ}$ & 95 & 2,4 & $18^{\circ}$ \\
\hline 7 & 23 & 1,3 & 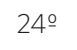 & 82 & 1,4 & $25^{\circ}$ & 17 & 1,3 & $23^{\circ}$ & 88 & 1,6 & 240 & 73 & 1,8 & $22^{\circ}$ \\
\hline 8 & 23 & 1,3 & $25^{\circ}$ & 83 & 1,4 & 240 & 10 & 0,8 & $25 \circ$ & 55 & 1,0 & $25^{\circ}$ & 30 & 0,7 & $25^{\circ}$ \\
\hline 9 & 60 & 3,4 & 140 & 205 & 3,5 & $12^{\circ}$ & 45 & 3,5 & $11^{\circ}$ & 228 & 4,2 & 90 & 203 & 5,1 & $8^{\circ}$ \\
\hline 10 & 108 & 6,1 & 40 & 359 & 6,2 & 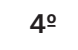 & 91 & 7,1 & 50 & 294 & 5,4 & 60 & 219 & 5,5 & 60 \\
\hline 11 & 56 & 3,2 & $16^{\circ}$ & 209 & 3,6 & $11^{\circ}$ & 26 & 2,0 & 190 & 159 & 2,9 & $16^{\circ}$ & 54 & 1,3 & $24^{\circ}$ \\
\hline 12 & 62 & 3,5 & $12 \circ$ & 127 & 2,2 & $21^{\circ}=$ & 49 & 3,8 & 10 & 112 & 2,1 & $211^{\circ}$ & 75 & 1,9 & $21^{\circ}=$ \\
\hline 13 & 76 & 4,3 & $10 \circ$ & 348 & 6,0 & 50 & 74 & 5,8 & 60 & 205 & 3,8 & 100 & 177 & 4,4 & $10 \circ$ \\
\hline
\end{tabular}




\begin{tabular}{|c|c|c|c|c|c|c|c|c|c|c|c|c|c|c|c|}
\hline \multicolumn{16}{|c|}{ Tabela 2. (cont.) } \\
\hline 14 & 52 & 2,9 & $18^{\circ}$ & 239 & 4,1 & 90 & 38 & 3,0 & $15^{\circ}$ & 176 & 3,2 & $14^{\circ}$ & 106 & 2,6 & 170 \\
\hline 15 & 109 & 6,1 & 30 & 322 & 5,5 & 60 & 94 & 7,4 & $4^{\circ}$ & 363 & 6,7 & 4o & 240 & 6,0 & $4 \%$ \\
\hline 16 & 50 & 2,8 & $20 \circ$ & 103 & 1,8 & $22^{\circ}$ & 19 & 1,5 & 210 & 102 & 1,9 & $22^{\circ}$ & 92 & 2,3 & 190 \\
\hline 17 & 104 & 5,9 & 50 & 290 & 5,0 & $8^{\circ}$ & 60 & 4,7 & $8^{\circ}$ & 273 & 5,0 & 70 & 239 & 6,0 & 5o \\
\hline 18 & 52 & 2,9 & $19 \circ$ & 133 & 2,3 & 20 & 18 & 1,4 & $22 ㅇ$ & 164 & 3,0 & 15 & 125 & 3,1 & $15 \circ$ \\
\hline 19 & 57 & 3,2 & 150 & 176 & 3,0 & $15^{\circ}$ & 27 & 2,1 & 180 & 157 & 2,9 & 170 & 132 & 3,3 & $14^{\circ}$ \\
\hline 20 & 69 & 3,9 & $11^{\circ}$ & 160 & 2,7 & $18^{\circ}$ & 25 & 2,0 & $20^{\circ}$ & 179 & 3,3 & 130 & 133 & 3,3 & 13 응 \\
\hline 21 & 77 & 4,3 & 9음 & 168 & 2,9 & $16^{\circ}$ & 36 & 2,8 & $16^{\circ}$ & 153 & 2,8 & $18^{\circ}$ & 139 & 3,5 & $12^{\circ}$ \\
\hline 22 & 62 & 3,5 & 130 & 143 & 2,5 & 190 & 34 & 2,7 & $17^{\circ}$ & 185 & 3,4 & $12^{\circ}$ & 150 & 3,7 & $11^{\circ}$ \\
\hline 23 & 45 & 2,5 & $21^{\circ}$ & 102 & 1,8 & $23^{\circ}$ & 17 & 1,3 & $24^{\circ}$ & 92 & 1,7 & $23^{\circ}$ & 66 & 1,6 & $23^{\circ}$ \\
\hline 24 & 92 & 5,2 & 70 & 230 & 4,0 & $10 \cong$ & 53 & 4,1 & 90 & 250 & 4,6 & $8^{\circ}$ & 198 & 4,9 & 90 \\
\hline 25 & 89 & 5,0 & 8ㅇ & 313 & 5,4 & 70 & 45 & 3,5 & $12^{\circ}$ & 333 & 6,1 & 5o & 212 & 5,3 & $7 \stackrel{0}{0}$ \\
\hline Total & 1.773 & 100 & - & 5.820 & 100 & - & 1.278 & 100 & - & 5.416 & 100 & - & 4.005 & 100 & - \\
\hline
\end{tabular}

Fonte: Elaboração própria

De acordo com as Regiões do País, os cinco desafios mais presentes estão descritos abaixo:

- Centro-oeste: desafios 4, 5, 15, 10 e 17.

- Nordeste: desafios 4, 5, 1, 10 e 13.

- Norte: desafios 4, 5, 1, 15 e 10.
- Sudeste: desafios 4, 5, 1, 15 e 25 .

- Sul: desafios 4, 5, 1, 15 e 17.

Observou-se que os desafios 4 e 5 destacaram-se em todas as Regiões do País. Os desafios 10 e 15 também foram encontrados em, pelo menos, três Regiões.

Tabela 3. Classificação dos desafios para a qualificação da AB nos municípios por Estrato - 2011

\begin{tabular}{|c|c|c|c|c|c|c|c|c|c|c|c|c|c|c|c|c|c|c|}
\hline \multirow{3}{*}{ 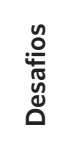 } & \multicolumn{18}{|c|}{ Estratos dos municípios } \\
\hline & \multicolumn{3}{|c|}{1} & \multicolumn{3}{|c|}{2} & \multicolumn{3}{|c|}{3} & \multicolumn{3}{|c|}{4} & \multicolumn{3}{|c|}{5} & \multicolumn{3}{|c|}{6} \\
\hline & № & $\%$ & C & № & $\%$ & C & № & $\%$ & C & № & $\%$ & C & № & $\%$ & C & № & $\%$ & C \\
\hline 1 & 478 & 8 & 3은 & 241 & 7 & $3 \circ$ & 165 & 6,6 & 3은 & 251 & 7 & $4 \stackrel{0}{\circ}$ & 85 & 6 & 5웅 & 46 & 7,6 & 3으 \\
\hline 2 & 258 & 4 & 10 & 126 & 3,7 & 110 & 81 & 3,3 & $12^{\circ}$ & 103 & 3 & $18^{\circ}$ & 33 & 2,3 & 190 & 11 & 1,8 & $22^{\circ}$ \\
\hline 3 & 209 & 3 & $16^{\circ}$ & 97 & 2,8 & $15^{\circ}$ & 74 & 3 & $17 \stackrel{0}{ }$ & 78 & 2 & $20^{\circ}$ & 20 & 1,4 & $25^{\circ}$ & 12 & 2 & $21^{\circ}$ \\
\hline 4 & 604 & 10 & $1 \stackrel{0}{ }$ & 348 & 10 & 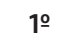 & 254 & 10 & $1 \stackrel{\circ}{\circ}$ & 357 & 10 & 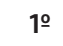 & 132 & 9,4 & $2^{\circ}$ & 65 & 11 & 1음 \\
\hline 5 & 533 & 9 & $2^{\circ}$ & 316 & 9,2 & 20 & 208 & 8,4 & 20 & 344 & 9 & 2 & 144 & 10 & $1 \stackrel{0}{0}$ & 57 & 9,4 & 2음 \\
\hline 6 & 179 & 3 & $18^{\circ}$ & 94 & 2,7 & 160 & 77 & 3,1 & $13^{\circ}$ & 75 & 2 & $21^{\circ}$ & 32 & 2,3 & 20 & 17 & 2,8 & $12^{\circ}$ \\
\hline 7 & 97 & 2 & $22^{\circ}$ & 44 & 1,3 & $25^{\circ}$ & 35 & 1,4 & $22^{\circ}$ & 62 & 2 & $24^{\circ}$ & 28 & 2 & $23^{\circ}$ & 10 & 1,7 & $23^{\circ}$ \\
\hline 8 & 48 & 1 & $25^{\circ}$ & 45 & 1,3 & $24^{\circ}$ & 23 & 0,9 & $25^{\circ}$ & 39 & 1 & $25^{\circ}$ & 24 & 1,7 & $24^{\circ}$ & 14 & 2,3 & $16^{\circ}$ \\
\hline 9 & 251 & 4 & $11 \stackrel{0}{ }$ & 137 & 4 & 10 & 77 & 3,1 & $14^{\circ}$ & 178 & 5 & 90 & 58 & 4,1 & 90 & 31 & 5,1 & 60 \\
\hline 10 & 396 & 6 & $4 \stackrel{0}{0}$ & 192 & 5,6 & $5^{\circ}$ & 161 & 6,5 & $5^{\circ}$ & 212 & 6 & 70 & 72 & 5,1 & 60 & 28 & 4,6 & 10 은 \\
\hline 11 & 77 & 1 & $24^{\circ}$ & 116 & 3,4 & $12^{\circ}$ & 116 & 4,7 & $8^{\circ}$ & 115 & 3 & $13^{\circ}$ & 53 & 3,8 & 110 & 16 & 2,6 & $15^{\circ}$ \\
\hline 12 & 98 & 2 & $21 \stackrel{0}{ }$ & 80 & 2,3 & $21 \stackrel{0}{ }$ & 76 & 3,1 & 16 & 96 & 3 & 19 & 49 & 3,5 & $13 ㅇ$ & 14 & 2,3 & $17 ㅇ$ \\
\hline
\end{tabular}




\begin{tabular}{|c|c|c|c|c|c|c|c|c|c|c|c|c|c|c|c|c|c|c|}
\hline \multicolumn{19}{|c|}{ Tabela 3. (cont.) } \\
\hline 13 & 359 & 6 & 50 & 176 & 5,1 & 70 & 114 & 4,6 & 9o & 167 & 4 & $10^{\circ}$ & 41 & 2,9 & $16^{\circ}$ & 10 & 1,7 & $24 \stackrel{\circ}{0}$ \\
\hline 14 & 237 & 4 & $12^{\circ}$ & 111 & 3,2 & $13 ㅇ$ & 85 & 3,4 & 110 & 116 & 3 & $12^{\circ}$ & 44 & 3,1 & $14^{\circ}$ & 4 & 0,7 & $25^{\circ}$ \\
\hline 15 & 352 & 6 & 60 & 215 & 6,2 & 40 & 164 & 6,6 & $4^{\circ}$ & 266 & 7 & 3은 & 86 & 6,1 & $4^{\circ}$ & 30 & 5 & 70 \\
\hline 16 & 132 & 2 & $20^{\circ}$ & 68 & 2 & $22^{\circ}$ & 35 & 1,4 & $23^{\circ}$ & 69 & 2 & $23^{\circ}$ & 29 & 2,1 & $22^{\circ}$ & 17 & 2,8 & 13 \\
\hline 17 & 326 & 5 & 70 & 176 & 5,1 & $8^{\circ}$ & 125 & 5 & 70 & 216 & 6 & 60 & 67 & 4,8 & $8^{\circ}$ & 39 & 6,4 & 5 은 \\
\hline 18 & 145 & 2 & 190 & 85 & 2,5 & $19 \circ$ & 54 & 2,2 & $21{ }^{\circ}$ & 105 & 3 & 170 & 55 & 3,9 & 100 & 30 & 5 & $8^{\circ}$ \\
\hline 19 & 214 & 3 & 140 & 85 & 2,5 & 20 & 72 & 2,9 & $18^{\circ}$ & 110 & 3 & 160 & 35 & 2,5 & 170 & 14 & 2,3 & $18^{\circ}$ \\
\hline 20 & 185 & 3 & $17^{\circ}$ & 101 & 2,9 & $14^{\circ}$ & 69 & 2,8 & $20^{\circ}$ & 111 & 3 & $15^{\circ}$ & 53 & 3,8 & $12^{\circ}$ & 27 & 4,5 & 110 \\
\hline 21 & 230 & 4 & 130 & 91 & 2,6 & $18^{\circ}$ & 70 & 2,8 & 190 & 118 & 3 & 110 & 30 & 2,1 & $21^{\circ}$ & 13 & 2,1 & $19 \circ$ \\
\hline 22 & 211 & 3 & $15^{\circ}$ & 93 & 2,7 & $17^{\circ}$ & 77 & 3,1 & $15^{\circ}$ & 112 & 3 & $14^{\circ}$ & 42 & 3 & $15^{\circ}$ & 17 & 2,8 & $14^{\circ}$ \\
\hline 23 & 83 & 1 & 230 & 64 & 1,9 & $23^{\circ}$ & 32 & 1,3 & $24^{\circ}$ & 72 & 2 & $22^{\circ}$ & 35 & 2,5 & $18^{\circ}$ & 13 & 2,1 & $20^{\circ}$ \\
\hline 24 & 263 & 4 & 90 & 156 & 4,5 & 9o & 96 & 3,9 & $10^{\circ}$ & 183 & 5 & $8^{\circ}$ & 72 & 5,1 & 70 & 29 & 4,8 & 90 \\
\hline 25 & 290 & 5 & $8^{\circ}$ & 184 & 5,3 & 60 & 144 & 5,8 & $6^{\circ}$ & 219 & 6 & $5^{\circ}$ & 89 & 6,3 & 30 & 41 & 6,8 & $4 \%$ \\
\hline Total & 6.256 & 100 & - & 3.441 & 100 & $100^{\circ}$ & 2.484 & 100 & - & 3.774 & 100 & - & 1.408 & 100 & - & 605 & 100 & - \\
\hline
\end{tabular}

Fonte: Elaboração própria

Considerando os Estratos dos municípios, os cinco desafios mais frequentes foram:

- Estrato 1: desafios 4, 5, 1, 10 e 13.

- Estrato 2: desafios 4, 5, 1, 15 e 10.

- Estrato 3: desafios 4, 5, 1, 15 e 10.

- Estrato 4: desafios 4, 5, 15, 1 e 25.

- Estrato 5: desafios 4, 5, 25, 15 e 1.

- Estrato 6: desafios 4, 5, 1, 25 e 17.

No caso dos Estratos, também verificamos que os desafios 4, 5 e 1 são recorrentes em todos. Constatou-se, ainda, que os desafios 10 e 15 continuam presentes em, pelo menos, três dos Estratos.

Comparando os desafios verificados nos níveis Nacional, Regional e dos Estratos, verificamos que os desafios 4 e 5 estiveram presentes em todos os agregados considerados e sempre atingiram as frequências mais altas. Logo, podemos dizer que os gestores consideram os processos de Monitoramento e Avaliação (M\&A) da Atenção Básica em Saúde (ABS) importantes para a qualificação da $A B$ e que destacam a necessidade de apoiar as equipes na análise e uso das informações em saúde, conferindo-se como instrumentos para auxiliar na tomada de decisão na esfera local.

Assim, a incorporação de atividades de M\&A na $A B$ faz todo sentido, já que elas podem auxiliar na definição dos problemas, reorientar as ações, fornecer elementos que permitam reconhecer as transformações ocorridas e mensurar o impacto das intervenções (AQUINO; MEDINA; ABDON, 2014).

Diversos autores (AQUINO; MEDINA; ABDON, 2014; CARVALHO, 2009; FELISBERTO, 2004) apontam a necessidade de o M\&A estar articulado às ações de gestão, como subsídio à formulação de políticas e ao processo decisório, devendo ser desenvolvido de forma permanente e sistemática. Explicam, ainda, que a preocupação com essa questão vem crescendo nos últimos anos, embora o M\&A ainda encontre-se defasado nos serviços, conforme observado neste estudo.

Fialho (2004) observou que uma das alternativas para ampliação da capacidade de 
análise e utilização da informação em saúde é estimular o seu uso pelas equipes a partir da atuação em serviço, demonstrando, com isso, possíveis abordagens. Para tanto, ressalta a necessidade de sensibilizar as equipes e estabelecer compromissos para uso, retorno e discussão da informação, além de adequações contínuas dos instrumentos utilizados. $\mathrm{O}$ autor afirma que o manejo das informações dentro dos próprios serviços de saúde pode apoiar a orientação/reorientação das práticas do cotidiano.

Os desafios 1, 10 e 15 também foram evidenciados nos três níveis de agregados, mesmo estando ausentes, um ou outro, em algumas Regiões e Estratos. Esses desafios reverberam em um problema pujante para os gestores, o financiamento, já que envolvem a informatização das UBS, assegurando o acesso à internet, o aperfeiçoamento da capacidade de gestão de pessoas da coordenação da ABS e as reformas das UBS próprias dos municípios.

Nas Regiões do País e nos Estratos, alguns novos desafios tonaram-se eminentes, podendo apontar problemas mais específicos quando nos aproximamos do nível mais descentralizado. Os desafios 13, 17 e 25 apontam para questões estruturantes para o sistema de saúde local, tais como a disponibilidade de equipamentos necessários para o desenvolvimento das ações de saúde pelas equipes, o Planejamento como ferramenta para a gestão e para a produção do cuidado junto às equipes e outros dispositivos da Rede de Atenção à Saúde (RAS), além da necessidade de construção de Linhas de Cuidado no município e/ou Região de Saúde.

Os desafios encontrados por Rocha et al. (2008) têm conexão com os verificados neste estudo. Segundo os autores, a pesquisa observou a necessidade de fortalecimento da capacidade formuladora do nível local, de alocação dos recursos na $\mathrm{AB}$, de desprecarização do trabalho, de fortalecimento da participação social, de qualificação do M\&A, para subsidiar a tomada de decisão, e de efetivação de RAS. Mesmo nos municípios considerados em estágios mais avançados de implantação e consolidação do PSF, permanece o desafio do sistema de saúde local de constituir-se em uma rede integrada de serviços.

Apontam como sugestões para a expansão e a consolidação do PSF a coerência entre o financiamento e os recursos aplicados na Atenção Básica, investimentos na adequação da estrutura dos serviços, escopo de ações de saúde diversificado (em função das reais necessidades das pessoas), estímulo à promoção da saúde e perfis de equipe compatíveis com conceitos ampliados de saúde (ROCHA ET AL., 2008).

Com relação à informatização, Facchini et al. (2014) verificaram, em 2005, que as UBS do País estavam bastante precárias nesse aspecto: em apenas $34 \%$ das UBS dos municípios havia computador. No que se refere à conexão com a internet, a situação era ainda pior. A partir de dados preliminares da avaliação externa do PMAQ, em 2001, observou-se um avanço no acesso ao computador, mas o acesso à internet permaneceu baixo.

Conforme observado por Santos et al., na pesquisa realizada em $\mathrm{BH}$, a incorporação de tecnologias de informação e a informatização no município impactaram positivamente a área assistencial. Esse processo caracterizou-se pela implantação de uma estrutura de gerenciamento da informação que ocorreu em um ambiente favorável à formulação de importantes políticas do sistema de saúde. Destaca-se, então, a priorização da gestão na implantação de inciativas dessa natureza.

Fausto, Mendonça e Giovanella (2013) comentam sobre alguns desafios que persistem na $\mathrm{AB}$ e destacam a diversidade das realidades dos territórios e as assimetrias regionais frente às normas nacionais. Afirmam que as realidades encontradas denotam a necessidade de modelos assistenciais mais flexíveis, em razão dos obstáculos que dificultam a garantia de ações e serviços de $A B$, e a conformação de redes de referência para a atenção especializada. 


\section{Considerações finais}

A partir deste estudo, podemos observar que 'problemas velhos' se tornaram 'novos desafios' (CAETANO; DAIN, 2002) para a AB. Diante dos resultados encontrados, podemos dizer que os desafios apontados pelos gestores municipais, em 2011, têm conexão com aqueles observados em outros estudos mencionados neste artigo.

Vimos que todos os gestores municipais consideram importante aperfeiçoar as atividades de monitoramento e avaliação da $\mathrm{AB}$ e que sinalizam a necessidade de auxiliar as equipes na análise e no manejo de informações para subsidiar a tomada de decisão no âmbito local. Outra grande parte dos gestores também evidenciou a necessidade de aprimorar os processos de gestão de pessoas no município, adequar a estrutura física das UBS, garantir a informatização das unidades

\section{Referências}

AQUINO, R.; MEDINA, M. G.; ABDON, C. Monitoramento e avaliação: reflexos visíveis na atenção primária à saúde no Brasil. In: SOUZA, M. F.; FRANCO, M. S.; MENDONÇA, A. V. M. (Org.). Saúde da família nos municípios brasileiros: os reflexos dos 20 anos no espelho do futuro. Campinas: Saberes editora, 2014.

BRASIL. Ministério da Saúde. Portaria GM n ${ }^{\circ} 2.488$, de 21 de outubro de 2011. Aprova a Política Nacional de Atenção Básica, estabelecendo a revisão de diretrizes e normas para a organização da atenção básica, para a Estratégia Saúde da Família (ESF) e o Programa de Agentes Comunitários de Saúde (PACS). Diário Oficial [da] União. Brasília, DF, 21 out. 2011a. Disponível em: <http://bvsms.saude.gov.br/bvs/saudelegis/gm/2011/ prt2488_21_10_2011.html>. Acesso em: 20 mar. 2014. com acesso a internet, desenvolver e estimular o Planejamento em Saúde e efetivar a RAS com ênfase na AB.

Observamos que alguns desafios foram evidenciados quando desagregamos do nível Nacional para analisar por região do País ou por estratos. Portanto, consideramos que as iniciativas já existentes que visam a enfrentar os desafios e qualificar a $\mathrm{AB}$ devem ser fortalecidas e outras devem ser desenvolvidas ou estimuladas com base nas realidades locais.

Por fim, consideramos que este estudo possibilitou reconhecer os desafios da $\mathrm{AB}$ sob a ótica dos gestores municipais, possibilitando empreender análises e comparações para o Brasil, Regiões e Estratos, podendo servir como instrumento para os gestores das três esferas de governo (municipal, estadual, federal) debaterem e refletirem soluções para o seu enfrentamento.
Ministério da Saúde. Secretaria de Atenção à Saúde. Departamento de Atenção Básica. Manual Instrutivo: Saúde Mais Perto de Você - Acesso e Qualidade. Programa Nacional de Melhoria do Acesso e da Qualidade da Atenção Básica. Brasília, 2012. Disponível em: <http://189.28.128.100/dab/ docs/publicacoes/geral/manual_instrutivo_pmaq_ site.pdf>. Acesso em: 1 mar. 2014.

Resolução no 439, de 7 de abril de 2011, do Conselho Nacional de Saúde. Sem descrição. Diário Oficial [da] União. Brasília, 7 abr. 2011b. Disponível em: <http://bvsms.saude.gov.br/bvs/saudelegis/ cns/2011/res0439_07_04_2011.html>. Acesso em: 1 maio 2014. 
CAMELO, S. H. H.; ANGERAMI, E. L. S. Formação de recursos humanos para a Estratégia de Saúde da Família. Revista Ciência, cuidado e saúde, Maringá, v. 7, n. 1, jan./mar. 2008, p. 45-52.

CAETANO, S.; DAIN, S. O Programa de saúde da família e a reestruturação da atenção básica à saúde nos grandes centros urbanos: velhos problemas, novos desafios. Physis: Revista de Saúde Coletiva, Rio de Janeiro, v. 12, n. 1. 2002, p. 11-21. Disponível em: <http:// www.scielo.br/scielo.php?script=sci_arttext\&pid =S0103-73312002000100002 >. Acesso em: 7 mar. 2014.

CARVALHO, A. L. B. Informação em saúde como ferramenta estratégica para a qualificação da gestão e o fortalecimento do controle social no SUS. Tempus Actas de Saúde Coletiva, Brasília, DF, v. 3, n. 3, jul./set. 2009, p. 16-30.

FACCHINI, L. A. et al. Os sentidos da pesquisa nos processos organizativos da Estratégia Saúde da Família. In: SOUZA, M. F. ; FRANCO, M. S.; MENDONÇA, A. V. M. (Org.). Saúde da família nos municípios brasileiros: os reflexos dos 20 anos no espelho do futuro. Campinas: Saberes Editora, 2014.

FAUSTO, M. C. R; MENDONÇA; M. H. M, GIOVANELLA, L. Experiências de avaliação da Atenção Básica no Brasil: notas para um debate. In: FAUSTO, M. C. R, FONSECA, H. M. S. (Org.). Rotas da atenção básica no Brasil: experiências do trabalho de campo PMAQ AB. Rio de Janeiro: Saberes Editora, 2013.

FELISBERTO, E. Monitoramento e avaliação na atenção básica: novos horizontes. Rev. Bras. Saude Mater. Infant., Recife, v. 4, n. 3, jul./set. 2004, p. 317-321. Disponível em: <http://www.scielo.br/pdf/rbsmi/ v4n3/a12v04n3.pdf >. Acesso em: 21 jun. 2014.

MERHY, E. E.; MALTA, D. C.; SANTOS, F. P. Desafios para os gestores do SUS, hoje: compreender os modelos de assistência à saúde no âmbito da reforma sanitária brasileira e a potência transformadora da gestão. In: FREESE, E. (Org.). Municípios: a gestão da mudança em saúde. Recife: UFPE, 2004.
PAIM, J. et al. O sistema de saúde brasileiro: história, avanços e desafios. The Lancet, London, maio 2011. Disponível em: <http://download.thelancet.com/flatcontentassets/pdfs/brazil/brazilporl.pdf $>$. Acesso em: 31 mar. 2014

PINTO, H. A; SOUSA, A. N. A ; FERLA, A. A. O Programa Nacional de Melhoria do Acesso e da Qualidade da Atenção Básica: várias faces de uma política inovadora. Saúde Debate, Rio de Janeiro. No prelo 2014.

ROCHA, P. M. et al. Avaliação do Programa Saúde da Família em municípios do Nordeste brasileiro: velhos e novos desafios. Cad. Saúde Pública, Rio de Janeiro, v. 24, supl. 1. 2008, p. 69-78. Disponível em: < http://www.scielo.br/scielo.php?pid=S0102$-311 X 2008001300012 \&$ script $=$ sci_arttext $>$. Acesso em: 14 jul. 2014.

SANTOS, A. F. et al. Estruturação da área de informação em saúde a partir da gerência de recursos informacionais: análise de experiência. Rev Panam Salud Pública. 2011, v. 29, n. 6, p. 409-415.

SAVASSI, L. C. M. Qualidade em serviços públicos: os desafios da atenção primária. Revista Brasileira de Medicina de Família e Comunidade, Rio de Janeiro, v. 7, n. 23, abr./jun. 2012, p. 69-74.

SOUSA, M. F. O Programa Saúde da Família no Brasil: análise do acesso à atenção básica. Rev. bras. enferm., Brasília, DF, v. 61, n. 2, mar./abr. 2008, p.153-8. Disponível em: <http://www.scielo.br/pdf/reben/ v6ln2/a02v6ln2>. Acesso em: 12 jan. 2014.

\section{Programa Saúde da Família no Brasil:}

Análise da desigualdade no acesso à atenção básica. Brasília: Departamento de Ciência da Informação e Documentação da Universidade de Brasília, DF, 2007. Disponível em: <http://www.scielo.br/pdf/reben/ v6ln2/a02v61n2>. Acesso em: 24 abr. 2014.

(Org.). Os Sinais vermelhos do PSF. São Paulo:

Hucitec, 2002.

A cor-agem do PSF. 2. ed. São Paulo: Huitec, 2003a. 
SOUSA, M. F.; MENDES, A. (Org.). Tempos radicais da saúde em São Paulo: a construção do SUS na maior cidade brasileira. São Paulo: Hucitec, 2003.

STARFIELD, B. Atenção primária: equilíbrio entre necessidades de saúde, serviços e tecnologia. Brasília, DF: UNESCO: Ministério da Saúde, 2002. Disponível em: <http://unesdoc.unesco.org/ images/0013/001308/130805por.pdf $>$. Acesso em: 12 jan. 2014.

TANAKA, O. U; RIBEIRO, E. L. Ações de saúde mental na atenção básica: caminho para ampliação da integralidade da atenção. Ciênc. saúde colet., Rio de Janeiro, v. 14, n. 2. 2009, p. 477-486.

TEIXEIRA, C. F. Enfoques Teórico-metodológicos do Planejamento em Saúde. In: Planejamento em

Saúde - Conceitos, Métodos e Experiências. Salvador: E DUFBA, 2010.

Recebido para publicação em outubro de 2014

Versão final em fevereiro de 2015

Conflito de interesse: inexistente

Suporte financeiro: não houve 